\title{
Conhecimento Especializado de Professores de Matemática (MTSK) na Web of Science até 2020
}

\author{
The Mathematics Teachers' Specialized Knowledge (MTSK) model in the Web of \\ Science until 2020
}

\author{
Jeferson Gomes Moriel Junior ${ }^{1}$
}

\begin{abstract}
Resumo
O objetivo deste trabalho é mapear a produção relativa ao marco teórico Mathematics Teachers' Specialized Knowledge na base de dados Web of Science. Para tanto, realizamos um pesquisa analítico-descritiva, de aproximação exploratório-bibliométrica, na base de dados referida. Encontramos um corpus de 37 trabalhos de 2013 a 2020 e analisamos: Indexação na Web of Science; Distribuição por ano e tipos de documentos; Autores; Instituições e agências de fomento; Países e idiomas; Breve análise de citações e Palavras-chave. Os resultados nos permitem concluir que a produção MTSK é colaborativa e internacional, envolvendo ao menos nove países da Europa e América Latina, num movimento em rede que contribui para a disseminação global e, de certo modo, configura-se como um teste de validação externa em diferentes culturas escolares e contextos de prática e formação docente.
\end{abstract}

Palavras-chave: MTSK; Web of Science; Mapeamento; Conhecimento Especializado de Professores de Matemática.

\begin{abstract}
The aim of this work is to map the production related to the Mathematics Teachers' Specialized Knowledge Model in the Web of Science database. For this, we carried out an analytical-descriptive research, with an exploratory-bibliometric approach. We found a corpus of 37 papers from 2013 to 2020 and analyzed: Indexing on the Web of Science; Distribution by year and types of documents; Authors; Development institutions and agencies; Countries and languages; brief Analysis of citations and Keywords. The results allow us to conclude that MTSK production is collaborative and global, involving at least 9 countries in Europe and Latin America, in a network movement that contributes to global dissemination and, in a way, is configured as an international validation test in different school cultures and contexts of teaching practice and training.
\end{abstract}

Keywords: MTSK; Web of Science; Review; Mathematics Teachers' Specialized Knowledge.

\section{Introdução}

A Educação Matemática tem analisado regularmente sua própria produção científica por meio de mapeamentos e estudos de síntese (como meta-análises qualitativas, revisões

\footnotetext{
Submetido em: 12/06/2020 - Aceito em: 24/09/2021 - Publicado em: 23/12/2021

1 Doutor em Educação em Ciências e Matemática pela Universidade Federal de Mato Grosso (UFMTREAMEC). Professor do Instituto Federal de Mato Grosso (IFMT), campus Cuiabá, Mato Grosso, Brasil. Email: jeferson.moriel@ifmt.edu.br. ORCID: https://orcid.org/0000-0002-2473-2957
} 
DOI: 10.20396/zet.v29i00.8660030

sistemáticas, estados da arte, dentre outros), para compreender os avanços e tendências nas diversas linhas da área. Isto tem possibilitado conhecer e (re)pensar caminhos e possibilidades de investigação visando melhorar a educação no país (Crecci et al., 2017; Fiorentini et al., 2016; Moriel Junior et al., 2019; Paula \& Cyrino, 2017).

Nesta perspectiva elaboramos o presente artigo como parte de um projeto mais amplo de mapeamento da produção sobre conhecimento especializado docente, na busca de sintetizar parte dos avanços científicos na educação matemática e aproximá-los da prática e formação docente (com fomento estadual e institucional). A temática escolhida está inserida no movimento do knowledge base, iniciado nos anos 1980, interessado em descrever os tipos de conhecimentos necessários ao professor. Desde então houve a geração de diferentes tipologias sobre conhecimento/saberes docentes e elas caminharam da perspectiva genérica para a especializada (Moriel Junior e Wielewski, 2017; Scheiner et al., 2019), ou seja, do conhecimento do professor de qualquer área para o do professor de disciplinas específicas.

Nesta trajetória, destacam-se com maior ênfase no primeiro grupo a tipologia de Shulman (1986) e de Tardif (2002), que somente nas obras citadas tem, respectivamente, mais de 25 e 12 mil citações na base do Google Scholar. Quando o foco é a disciplina de Matemática, destaca-se o modelo Mathematics Knowledge for Teaching de Ball et al. (2008) com quase 7 mil citações e, mais recentemente, o Mathematics Teachers' Specialized Knowledge - MTSK de Carrillo et al. (2014) com reconhecimento internacional na área (Kilpatrick e Spangler, 2015), mas escassez de estudos síntese para compreensão da sua produção. Diante disso, o objetivo deste artigo é mapear a produção relativa ao Mathematics Teachers' Specialized Knowledge - MTSK na base de dados Web of Science até o ano de 2020.

\section{O marco teórico MTSK}

O Mathematics Teachers' Specialized Knowledge - MTSK é um modelo teórico que descreve o conhecimento profissional específico e especializado que possui (ou deve possuir) um professor para ensinar matemática (Carrillo et al., 2014; Carrillo-Yañez et al., 2018). Ele foi desenvolvido considerando as principais caracterizações, tipologias e modelos feitos por pesquisadores da área até o momento e avançando em relação aos limites neles detectados (Escudero et al., 2012; Kilpatrick \& Spangler, 2015; Montes et al., 2013; Scheiner et al., 2019). É configurado em formato hexagonal com dois domínios e seis subdomínios (Figura 1). No centro estão as crenças de professores sobre a Matemática, seu ensino e aprendizagem, pois permeiam os subdomínios e dão sentido às suas ações. A seguir, descrevemos cada subdomínio com base em Carrillo et al. (2014) e Carrillo-Yañez et al. (2018). 


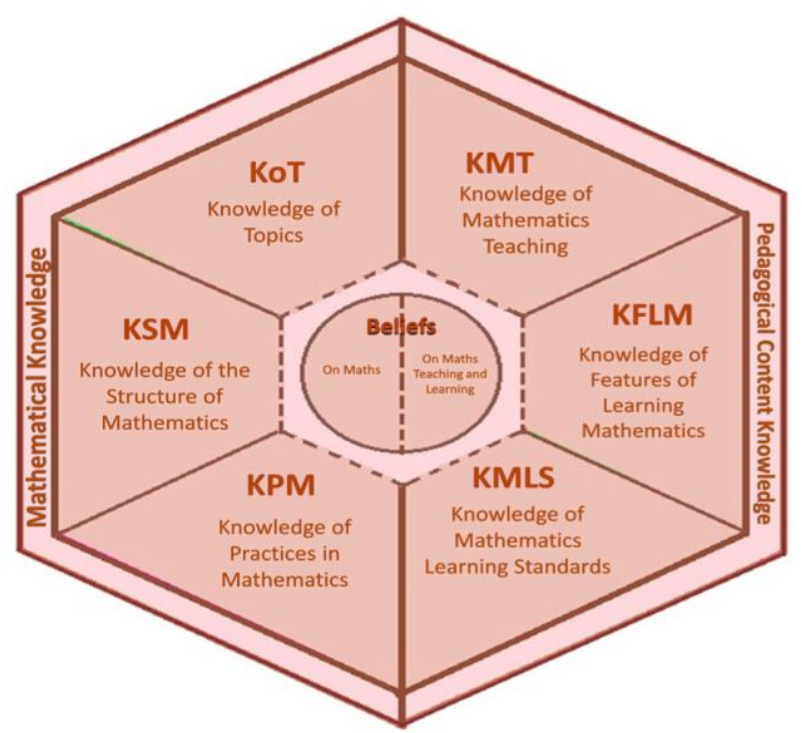

Figura 1 - Domínios e subdomínios do MTSK. Fonte: Carrillo-Yañez et al. (2018)

No domínio do Mathematical knowledge, há o subdomínio Knowledge of Topics (KoT) que abarca conteúdos matemáticos a serem ensinados (e sua fundamentação conceitual profunda) e inclui definições, interpretações e propriedades de conceitos, uma ou mais demonstrações de um tópico específico, justificativas de procedimentos algorítmicos, exemplos e contraexemplos, modelos realísticos, situações de aplicação e usos extra matemáticos. São exemplos, conhecer sistemas representacionais sobre divisão (Policastro \& Ribeiro, 2021) ou diferentes algoritmos (e procedimentos alternativos) para dividir frações e suas justificativas (Moriel Junior et al., 2019). Também inclui conexões intraconceituais, como é o caso dos conceitos de relação e de função de $1^{\circ}$ grau em que a definição de um é determinada pelo outro (Vasco et al., 2017). No Knowledge of the structure of mathematics (KSM) estão as conexões interconceituais (entre tópicos avançados e elementares, prévios e futuros, de diferentes áreas matemáticas etc., exceto as intraconceituais previstas em KoT) que permitem reconhecer certas estruturas da Matemática, bem como, vê-la como um sistema de elementos integrados. São exemplos conhecer que os conceitos de limite, derivada e integral tem em comum um processo de subdivisão infinita ou que o conceito de raiz quadrada pode ser utilizado para calcular as raízes de uma equação de $2^{\circ}$ grau (Courant \& Robbins, 1996). O Knowledge of practices in mathematics (KPM) inclui maneiras de proceder em Matemática, incluindo modos de criar ou produzir na área (conhecimento sintático), aspectos da comunicação matemática, elementos que estruturam uma demonstração, modos de provar e definir, de selecionar representações, de argumentar, de generalizar a partir de padrões e regularidades (Campos-Cano e Flores-Medrano, 2019).

Quanto aos subdomínios do Pedagogical contente knowledge, há o Knowledge of features of learning mathematics (KFLM) inclui como os alunos aprendem os conteúdos matemáticos (modelos e teorias formais ou pessoais), as características desse processo de compreensão, erros comuns e suas fontes prováveis, dificuldades, obstáculos e a linguagem normalmente usada pelos aprendizes ao lidar com cada conceito. Por exemplo, conhecer a 
teoria APOS (Arnon et al., 2014) ou as seis etapas de aprendizagem (Dienes, 1975) sobre o desenvolvimento cognitivo de um estudante em Matemática, conhecer erros comuns de estudantes ao lidarem com frações (Ashlock, 2006; Bayoud, 2011) ou ainda, conhecer os interesses e expectativas discentes para aprendizagem matemática (Kaur, 2008). O Knowledge of mathematics teaching (KMT) que diz respeito a materiais, recursos, modos de apresentar um conteúdo e suas respectivas características (limitações ou potencialidades existentes em si mesmos) que permitam ao professor optar por uma estratégia para ensinar determinado conteúdo (incluindo organizar uma série de exemplos ou criar analogias e metáforas). Por exemplo, conhecer a estratégia de ensinar frações utilizando uma figura geométrica (circular ou retangular, por exemplo) ou um modelo (como pizzas ou chocolates) e saber que isto é (mais) adequado para desenvolver a interpretação parte-todo (Moreira \& Ferreira, 2008). Também inclui o conhecimento (formal ou pessoal) de elementos teóricos sobre o ensino de Matemática como, por exemplo, tipos de explicações instrucionais (Charalambous et al., 2011) ou abordagens baseadas em resolução de problemas ou modelagem (Biembengut \& Hein, 2007; Krulik \& Reys, 1998). O Knowledge of mathematics learning standards (KMLS) se refere a especificações curriculares envolvendo as diretrizes nas diversas etapas educativas sobre conteúdos e competências (conceituais, procedimentais, atitudinais e de raciocínio matemático), bem como as formas avaliativas e de progressão de um ano para outro.

A construção de conhecimentos especializados provém tanto de fontes acadêmicocientíficas - como livros de conteúdo e didáticos, revistas e artigos científicos, meta-análises, parâmetros curriculares, dentre outros (Becker, 2019; Courant \& Robbins, 1996; Petit et al., 2010) -, quanto de fontes profissionais da cultura escolar, como relatos de experiência e diálogo com outros docentes.

\section{Metodologia}

Esta é uma pesquisa analítico-descritiva, de aproximação exploratório-bibliométrica, dada a finalidade acompanhar e mensurar o crescimento e desenvolvimento da produção científica numa determinada área da Educação Matemática relativa ao MTSK (Araújo, 2016). Neste sentido, "buscamos não apenas quantificar e constatar, mas também atribuir [algum] sentido [possível] aos dados, qualificando-os para que possam ter melhor uso [...] em contextos mais abrangentes, regionais, nacionais ou mundiais" (Santos e Kobashi, 2009, p. 159, grifo nosso), por educadores matemáticos, investigadores ou grupos de pesquisa. Este trabalho se insere nos esforços do grupo de pesquisa Teachers' Specialized Knowledge Research Group ${ }^{2}$, coordenado pelo autor junto ao IFMT, para acompanhar os avanços científicos sobre o tema.

\footnotetext{
${ }^{2}$ Acesso em http://dgp.cnpq.br/dgp/espelhogrupo/7032020622091895
} 
DOI: $10.20396 /$ zet.v29i00.8660030

A fonte de dados é a base científica Web of Science (WoS), que foi acessada por meio do Portal Periódicos ${ }^{3}$ da Coordenação de Aperfeiçoamento de Pessoal de Nível Superior (CAPES) utilizando o "Acesso remoto via CAFe" pelo perfil do autor como docente do IFMT. O tipo de informação que analisamos é o de registros bibliográficos da produção científica (livros, periódicos, artigos etc.) indexada na referida base. O universo de informação disponível no dia das buscas (6 de junho de 2020) totalizava 73.217.951 registros. A busca foi atualizada em 5 de agosto de 2021.

Para selecionar o material de análise, utilizamos o termo "MTSK" nas buscas na fonte "Principal Coleção do Web of Science", abrangendo "Todos os campos" e todos os anos (1945 a 2020). A segunda etapa foi realizada com os 66 registros resultantes, nos quais aplicamos o critério de inclusão (conter no título os termos specialized ou knowledge ou mathematics) e o de exclusão (ser de área diversa à educação matemática, como bioquímica, biofísica, biotecnologia, microbiologia, engenharia, computação), chegando a 32 itens. Na terceira etapa, refinamos a busca considerando os artigos que citavam estes 32 e identificamos mais quatro que atendiam os critérios, totalizando 37 registros que compuseram o corpus de análise (Apêndice). Para a coleta de dados, salvamos uma lista dentro da WoS com todos os registros do corpus (nosso banco de dados), exportamos para o software Endnote, para arquivos de texto e de planilha eletrônica para posterior análise.

$\mathrm{Na}$ análise de dados, utilizamos as ferramentas da WoS intituladas "Análise de resultados" e "Relatório de citações" para gerar dados consolidados e interativos que permitiam o detalhamento das informações encontradas sobre a produção. A partir deles, realizamos leituras sucessivas e novos agrupamentos, quando necessário, por similaridade de conteúdo e frequência de dados. Dadas as inconsistências de registro inerentes a este tipo de base de dados (algo comum em todas as bases científicas), foi feito sempre que necessário a padronização/limpeza de nomes de autores, instituições etc. Na análise utilizamos Estatística Descritiva e análise de conteúdo para explorar os títulos e os resumos do corpus. Deste modo, a produção MTSK foi investigada abrangendo as seguintes categorias: 1. Indexação na Web of Science (Áreas de pesquisa associadas); 2. Distribuição da produção por ano e tipos de documentos; 3. Autores envolvidos; 4. Instituições e agências de fomento envolvidas; 5. Países e idiomas; 6. Breve análise de citações; 7. Palavras-chave.

\section{Resultados}

Nossos resultados indicam 37 produções MTSK de 2013 até 2020, as quais discutimos a seguir, segundo as categorias de análise. Esclarecemos que mantivemos os termos no idioma original da base de dados (inglês) quando se tratou de nomes de categorias, de áreas, de eventos, de periódicos e de palavras-chave utilizadas na Web of Science.

Indexação da produção na Web of Science (Áreas de pesquisa)

\footnotetext{
${ }^{3}$ Acesso em http://periodicos.capes.gov.br
} 
DOI: $10.20396 /$ zet.v29i00.8660030

A produção científica sobre MTSK nesta base está integralmente indexada a "Área de pesquisa" nomeada Education Educational Research e minoritariamente à Mathematics (Tabela 1). Saber quais são as áreas nas quais os trabalhos estão classificados nesta base de dados serve como critério facilitador em buscas futuras para os interessados em identificar estudos nesta temática e para atualização futura dos mapeamentos.

Tabela 1 - Áreas de pesquisa e Categorias do Web of Science da produção MTSK (2013 a 2020)

\begin{tabular}{lc}
\hline Classificação & Artigos por \\
\hline Education Educational Research & Área de pesquisa \\
Mathematics & $37(100 \%)$ \\
\hline
\end{tabular}

Fonte: Dados organizados pelo autor.

\section{Distribuição da produção por ano e tipos de documentos}

A distribuição da produção MTSK por ano indica que as primeiras publicações indexadas na Web of Science ocorreram em 2013 (Tabela 2). Trata-se de três artigos publicados em anais do $17^{\circ}$ Simpósio anual da Sociedade Espanhola de Investigação em Educação Matemática (SEIEM) realizado em Bilbao, Espanha. Desde então, a produção nunca foi inferior a três trabalhos por ano nesta base, tendo atingido a máxima quantidade em 2018, ano que representa quase um quarto do total do período (2013 a 2020) e um acumulado de $78 \%$ do corpus. Em nosso corpus há apenas dois tipos de trabalhos, sendo que os artigos publicados em eventos predominam ligeiramente (54\%) frente aos publicados em periódicos $(46 \%)$.

Tabela 2 - Distribuição da produção MTSK por ano na Web of Science (2013 a 2020)

\begin{tabular}{lccccccccc}
\hline Tipo de trabalho & $\mathbf{2 0 1 3}$ & $\mathbf{2 0 1 4}$ & $\mathbf{2 0 1 5}$ & $\mathbf{2 0 1 6}$ & $\mathbf{2 0 1 7}$ & $\mathbf{2 0 1 8}$ & $\mathbf{2 0 1 9}$ & $\mathbf{2 0 2 0}$ & Total \\
\hline Artigo em evento & 3 & 3 & 4 & 3 & 3 & 4 & - & - & 20 \\
Artigo em periódico & - & - & 2 & 1 & - & 6 & 3 & 5 & 17 \\
\hline Total no ano & 3 & 3 & 6 & 4 & 3 & 10 & 3 & 5 & 37 \\
Total acumulado & 3 & 6 & 12 & 16 & 19 & 29 & 32 & 37 & - \\
\hline
\end{tabular}

Fonte: Dados Web of Science organizados pelo autor.

Levantamentos prévios (Moriel Junior e Duarte, 2020) identificam que a primeira produção ocorreu em 2012 (Escudero et al., 2012), fora do arco temporal identificado na WoS. Podemos dizer, então, que uma fase de constituição do MTSK ocorreu entre 2012 e 2014, culminando na publicação do livro intitulado Un marco teórico para el Conocimiento especializado del Profesor de Matemáticas (Carrillo et al., 2014). Nele foi apresentado a conceptualização do modelo e sua estrutura de crenças, domínios e subdomínios, obtidos até aquele momento. Na WoS, também há um trabalho em que os criadores apresentam o MTSK (Carrillo-Yañez et al., 2018) com alguns refinamentos (em termos de categorias, por exemplo) fruto do contínuo desenvolvimento e validação teórico-empírica do modelo.

\footnotetext{
${ }^{4}$ Tradução de Proceedings Paper.
} 
A produção aqui identificada é muito importante por ser um conjunto de trabalhos indexados em uma base de alto impacto e relevância científicos. Trata-se de uma parte seleta de toda a produção MTSK disponível em diversas outras bases, como a do Google Scholar (Moriel Junior \& Duarte, 2020). Estudos comparativos e integrativos da produção em diferentes bases serão foco de trabalhos posteriores.

\section{Autores}

Identificamos 45 pesquisadores envolvidos com a produção investigada. Um terço deles (16), aproximadamente, tem mais de uma publicação indexada na WoS (Tabela 3) e esses autores juntos participam de 31 trabalhos, isto é, $86 \%$ de toda a produção encontrada. Isso sugere um cenário colaborativo entre investigadores, e de internacionalização dado que o grupo que mais publicou tem produzido com os demais autores, de diversas partes do mundo (os dois terços com apenas uma publicação).

Tabela 3 - Autores e participação na produção MTSK na Web of Science (2013 a 2020)

\begin{tabular}{|c|c|c|c|}
\hline Autores $^{5}$ & País ${ }^{6}$ & $\mathbf{N}^{0}$ de trabalhos & Percentual \\
\hline Carrillo-Yanez, José & Espanha & 18 & $48,6 \%$ \\
\hline Contreras, Luis Carlos & Espanha & 10 & $27,0 \%$ \\
\hline Montes, Miguel & Espanha & 10 & $27,0 \%$ \\
\hline Climent-Rodriguez, Nuria & Espanha & 8 & $21,6 \%$ \\
\hline Munoz-Catalan, M. Cinta & Espanha & 7 & $18,9 \%$ \\
\hline Escudero-Avila, Dinazar & México & 6 & $16,2 \%$ \\
\hline Flores-Medrano, Eric & México & 6 & $16,2 \%$ \\
\hline Aguilar Gonzalez, Alvaro & Espanha & 5 & $13,5 \%$ \\
\hline Liñán Garcia, Maria Del Mar & Espanha & 5 & $13,5 \%$ \\
\hline Zakaryan, Diana & Chile & 5 & $13,5 \%$ \\
\hline Espinoza-Vasquez, Gonzalo & Chile & 3 & $8,1 \%$ \\
\hline Vasco, Diana & Equador & 3 & $8,1 \%$ \\
\hline Barrera, Victor & Espanha & 2 & $5,4 \%$ \\
\hline Flores, Pablo & Espanha & 2 & $5,4 \%$ \\
\hline Pascual, Maria Isabel & Espanha & 2 & $5,4 \%$ \\
\hline Ribeiro, Miguel & Brasil & 2 & $5,4 \%$ \\
\hline
\end{tabular}

Arteaga-Martinez, Blanca (Espanha); Badillo, Edelmira (Espanha); Belmonte, Juan Miguel (Espanha); Borromeo-Ferri, Rita (Alemanha); Caceres, Maria José (Espanha); Cardenas, Janeth Amparo (Espanha); Carreno, Enrique (Peru); Carvajal, Christian Alfaro (Costa Rica); Codes, Myrian (Espanha); Delgado-Rebolledo, Rosa (Chile); Escudero-Dominguez, Ana (Espanha); Fernandez, Ceneida (Espanha); Ferretti, Federica (Itália); Prieto Gonzalez, Alba (Espanha); Huincahue, Jaime (Chile); Leon, Aurora Fernandez (Espanha); Martin, Juan Pedro (Espanha); Mena-Lorca, Jaime (Chile); Montoya-Delgadillo, Elizabeth (Chile); Moriel Junior, Jeferson Gomes (Brasil); Oliveros, Ingrid (Colombia); Pizarro, Noemi (Chile); Rodriguez; Muniz, Luis Jose (Espanha); Rojas, Nielka (Chile); Soto, Gabriel Valverde (Costa Rica);Verdugo-Hernandez, Paula (Chile); Lira, Marcela Muñoz (Chile).

Fonte: Dados Web of Science organizados pelo autor.

\footnotetext{
${ }^{5}$ Formato "Sobrenome, Nome".

${ }^{6}$ País de vínculo institucional do autor conforme WoS.
} 
DOI: $10.20396 /$ zet.v29i00.8660030

A colaboração entre pesquisadores se expressa também quando analisamos o número de autores por produção. Há apenas três trabalhos publicados individualmente (Tabela 4), reforçando assim, os resultados anteriores. As características aqui identificadas parecem contribuir para o avanço na disseminação global e, de certo modo, configura um teste de validação internacional do MTSK em diferentes culturas escolares e contextos de prática e formação docente. Estudos em outras bases estão sendo realizados para ampliar a compreensão deste panorama.

Tabela 4 - Quantidade de autores por produção MTSK por ano na Web of Science (2013 a 2020)

\begin{tabular}{cccccccccc}
\hline Autores & $\mathbf{2 0 1 3}$ & $\mathbf{2 0 1 4}$ & $\mathbf{2 0 1 5}$ & $\mathbf{2 0 1 6}$ & $\mathbf{2 0 1 7}$ & $\mathbf{2 0 1 8}$ & $\mathbf{2 0 1 9}$ & $\mathbf{2 0 2 0}^{\mathbf{1}}$ & Total \\
\hline Um autor & - & 1 & - & - & - & - & - & 2 & 3 \\
\hline Dois autores & 1 & 2 & 1 & - & 1 & 3 & 3 & 1 & 12 \\
\hline Três autores & 2 & - & 1 & 1 & 2 & 4 & - & 2 & 12 \\
\hline Mais de três autores & - & - & 4 & 3 & - & 3 & - & - & 10 \\
\hline Total de trabalhos & 3 & 3 & 6 & 4 & 3 & 10 & 3 & 5 & 37 \\
\hline
\end{tabular}

Fonte: Dados Web of Science organizados pelo autor.

\section{Instituições e agências de fomento envolvidas}

Nossos resultados mostram um total de 27 instituições envolvidas na produção MTSK na WoS (Tabela 5). Nove delas têm mais de uma publicação e estão presentes em praticamente todas as demais (32 de 37). A instituição com mais publicações é a Universidade de Huelva, Espanha, algo que parece condizente com a história recente já que o marco teórico foi ali desenvolvido pelo grupo de pesquisa $\mathrm{SIDM}^{7}$ coordenado pelo Prof. Dr. José Carrillo.

As teses doutorais de membros do SIDM (Nielka Rojas, Jeferson Moriel-Junior, Miguel A. Montes, Eric Flores, Dinazar Escudero, Diana Vasco, Álvaro Aguilar, Mar Liñán) permitiram desenvolver o modelo, particularmente seu sistema de categorias e indicadores, no qual também participaram os demais membros (Carrillo, 2017, p. 8, tradução nossa).

Tabela 5 - Instituições envolvidas na produção MTSK na Web of Science (2013 a 2020)

\begin{tabular}{|c|c|c|}
\hline Instituições & $\mathbf{N}^{\circ}$ de trabalhos & Percentual \\
\hline Universidade de Huelva & 27 & $72,9 \%$ \\
\hline Universidade de Sevilha & 10 & $27,0 \%$ \\
\hline Pontifícia Universidade Católica Valparaiso & 7 & $18,9 \%$ \\
\hline Universidade de Oviedo & 3 & $8,1 \%$ \\
\hline Universidade Técnica Estatal de Quevedo & 3 & $8,1 \%$ \\
\hline Universidade Cardenal Spinola Ceu & 2 & $5,4 \%$ \\
\hline Universidade de Granada & 2 & $5,4 \%$ \\
\hline Universidade Autônoma de Puebla & 2 & $5,4 \%$ \\
\hline $\begin{array}{ll}\text { Universidade Estadual de Campinas } & \text { Brasil } \\
\end{array}$ & 2 & $5,4 \%$ \\
\hline $\begin{array}{l}\text { Instituto Federal de Mato Grosso - IFMT (Brasil), Universidade Autônoma } \\
\text { de Barcelona (Espanha), Universidade Católica de Maule (Chile), } \\
\text { Universidade Católica do Norte (Chile), Universidade Complutense de Madri }\end{array}$ & 1 & $2,7 \%$ \\
\hline
\end{tabular}

\footnotetext{
${ }^{7}$ Intitulado Seminário de Investigadores em Didáctica de las Matemáticas

${ }^{8}$ País de vínculo institucional do autor conforme WoS.
} 
(Espanha), Universidade de Alcala (Espanha), Universidade de Alicante (Espanha), Universidade de Costa Rica (Costa Rica), Universidade de Extremadura (Espanha), Universidade de Piura (Peru), Universidade de Salamanca (Espanha), Universidade Livre de Bolzano (Itália), Universidade Kassel (Alemanha) e Universidade Metropolitana de Ciências da Educação UMCE (Chile)

Fonte: Dados Web of Science organizados pelo autor.

Quanto ao apoio de agências de fomento a pesquisas, constam 5 registros explícitos na base de dados, quais sejam: Comissão Nacional de Investigação Científica e Tecnológica CONICYT do Chile, Ministério da Economia e Competitividade da Espanha, Centro de Pesquisa COIDESO da Universidade de Huelva na Espanha e Subsecretaria de Educação Superior PRODEP do México. Estes dados estão alinhados aos anteriores, na medida em que esses três países são os que possuem maior número de instituições com produção nesta base.

Estes resultados desta seção convergem com os anteriores, tanto no aspecto colaborativo da produção MTSK, quanto na presença de autores que mais produzem vinculados às respectivas Instituições. Os dados por países, a seguir, também convergem.

\section{Países e idiomas}

A produção MTSK advém de nove países na WoS (Tabela 6). Dos trabalhos encontrados nesta base, praticamente todos (32 de 37) têm autores vinculados institucionalmente na Espanha. Isto também é um reflexo de onde o marco teórico foi desenvolvido e a partir dali disseminado. Dos nove países encontrados, apenas a Alemanha não possui membros ou colaboradores da Rede Ibero-americana MTSK ${ }^{9}$. Isto significa interesse e disseminação dentro da América Latina e em países da Europa. A continuidade do estudo em outras bases de dados pode ajudar a esclarecer não somente se há mais produções nestes países, como também desvelar a existência de trabalhos em outras regiões do mundo.

Tabela 6 - Vínculo institucional da produção MTSK por países na Web of Science (2013 a 2020)

\begin{tabular}{lccccccccc}
\hline País & Espanha & Chile & Brasil & Equador & México & Peru & $\begin{array}{c}\text { Costa } \\
\text { Rica }\end{array}$ & Itália & Alemanha \\
\hline Qtde. de trabalhos & 32 & 9 & 3 & 3 & 2 & 1 & 1 & 1 & 1 \\
associados & $86,4 \%$ & $24.3 \%$ & $8,1 \%$ & $8,1 \%$ & $5,4 \%$ & $2,7 \%$ & $2,7 \%$ & $2,7 \%$ & $2,7 \%$ \\
\hline
\end{tabular}

Fonte: Dados Web of Science organizados pelo autor.

É sabido que a ciência internacional de impacto se comunica pelo idioma inglês. Entretanto, na produção MTSK predomina o idioma espanhol (Tabela 7). Embora possa parecer algo negativo em termos de disseminação, trata-se do segundo idioma mais importante de comunicação internacional. Vemos isso como um aspecto facilitador para disseminação em países da América Latina (Tabela 6) onde predomina tal idioma e também é

\footnotetext{
${ }^{9}$ Configurada em 2016, atualmente tem 129 membros de 10 países (Argentina, Brasil, Chile, Colômbia, Costa Rica, Equador, Espanha, México, Peru e Venezuela), colaboradores de Portugal e Itália e recebe apoio da Asociación Universitaria Iberoamericana de Postgrado - AUIP.
} 
DOI: $10.20396 /$ zet.v29i00.8660030

familiar a países de língua portuguesa como Brasil e Portugal, pertencentes a Rede Iberoamericana MTSK.

Tabela 7 - Distribuição da produção MTSK por ano na Web of Science (2013 a 2020)

\begin{tabular}{lcccc}
\hline Idioma & Espanhol & Inglês & Português & Total \\
\hline Qtde. de trabalhos & 26 & 9 & 1 & 37 \\
\hline
\end{tabular}

Fonte: Dados Web of Science organizados pelo autor.

De todo modo, constatamos que o artigo do corpus de maior impacto está em inglês, conforme detalhamos a seguir.

\section{Breve análise de citações}

Quanto à disseminação dos trabalhos segundo a WoS, o conjunto de produções do corpus investigado possui na data da coleta de dados um $h$-index ${ }^{10}$ igual a 4 , média de 10,63 citações/ano e 2,3 citações/item, soma do número de citações igual a 85 e há 66 artigos que fizeram a citação de trabalhos do corpus. O trabalho mais citado, com média de 7,25 citações por ano, foi publicado em 2018 na revista Research In Mathematics Education intitulado The mathematics teacher's specialised knowledge (MTSK) model (Carrillo-Yañez et al., 2018). Isto faz sentido, pois este artigo é particularmente importante uma vez que os criadores do MTSK fazem a apresentação formal do marco teórico em um periódico científico de alto impacto na área $(h$-index $=18$, SJR $2020=0,96$; CiteScore $2020=2,1$; Q1 Scopus; Q2 JCI WoS).

\section{Palavras-chave}

Todas as palavras-chave, títulos e resumos analisados estavam em língua inglesa, por isso mantivemos este idioma nos resultados, exceto quando apresentamos conteúdos matemáticos, dada a equivalência entre idiomas. A análise das produções MTSK indicou 146 palavras-chave no total (aproximadamente 4 por trabalho), que resultaram em 78 termos após análise das repetições e similaridade. A partir deste grupo, foi possível identificar 16 palavras-chave associadas a tópicos matemáticos, apresentados a seguir em ordem decrescente: conceito de função (3), posições relativas de retas (3), infinito, quadriláteros (2), geometria, aritmética (1), funções, números racionais, segmento de reta, análise real, séries, subtração, sistemas de equações lineares, álgebra linear, matrizes e números.

Os demais termos (62) descrevem os aspectos teórico-metodológicos das produções, os quais apresentamos na sigla original em ordem decrescente de frequência: Mathematics teacher's specialised knowledge (16), MTSK (15), Professional knowledge (8), Prospective teachers of mathematics (8), Mathematics teachers (3), Initial training of teachers, Mathematical Knowledge for Teaching MKT, Video analysis, Teacher (2), Analogies, Case

\footnotetext{
${ }^{10} \mathrm{O}$ índice h é baseado em uma lista de publicações classificadas em ordem decrescente pelo Times Cited no WoS. O índice h igual a 3 significa que no corpus existem 3 artigos com 3 ou mais citações. Para mais detalhes, recomendamos acessar https://clarivate.libguides.com/woscc/citationreport.
} 
DOI: $10.20396 /$ zet.v29i00.8660030

study, Knowledge of Mathematics Topics KoT, Pedagogical Content Knowledge, Pre-service teachers, Prospective Primary teachers, University lecturer, Activity design (1), Analysis scenarios, Conceptions of teaching-learning mathematics, Curriculum, Definition, Didactic, Early childhood education, Evaluation, Feedback, Formative evaluation, High school student, High school teacher, Knowledge about mathematics learning, Knowledge indications, Knowledge of practices in mathematics KPM, Links and differences Mathematical modelling, Mathematical proof, Mathematics lecturer, Mathematics, Metacognition, MWS (se referindo ao Mathematical Working Space), Narrative, Online forum, Online teacher education, Opportunities, Pedagogical practice, Pre-service primary teacher education, Problem solving, Reflection, Research methodology, Secondary school, Secondary teacher knowledge, Secondary, Self-questions, Specialised knowledge relations, Strength, Teachers' practices, Teaching of modeling model, Teaching, TEDS-M, Theoretical perspectives, Time measurement instrument, Time, To classify, To define, Trainee teachers mathematics knowledge e Weakness.

Os elementos encontrados nas palavras-chave sugerem que os estudos com MTSK têm utilizado uma gama considerável de aproximações metodológicas para investigar a prática e a formação docente (incluindo o ensino a distância), contemplando conteúdos dos diversos níveis da educação (desde a infantil até a universidade) e os dois domínios do MTSK, didático do conteúdo e matemático, com destaque para o subdomínio Knowledge of Topics e Knowledge of Pratices in Mathematics.

Estes resultados são uma primeira aproximação para compreendermos as tendências temáticas e teórico-metodológicas da produção MTSK. Estas palavras-chave servirão em estudos futuros para análise de similares com outras bases, visando a integração dos mapeamentos. Posteriormente, eles terão papel auxiliar no momento da análise de conteúdo que aprofunda a perspectiva qualitativa, incluindo leituras sucessivas dos resumos e textos.

\section{Considerações finais}

Neste artigo mapeamos a produção relativa ao marco teórico Mathematics Teachers' Specialized Knowledge - MTSK na base de dados Web of Science (WoS), cujos resultados são:

- Há 37 trabalhos sobre conhecimento especializado de professores de Matemática na WoS, todos indexados na categoria/área Education Educational Research;

- Há produção de 2013 até 2020 no WoS, no formato de artigos em periódicos e anais de eventos (leve predomínio deste último), com pelo menos 3 trabalhos/ano e pico de 10 em 2018, predominantemente em idioma Espanhol;

- 44 pesquisadores (em 9 países ibero-americanos e 27 instituições estão envolvidos colaborativamente com pesquisa MTSK, de modo que há um núcleo de 16 deles (de 4 países e 9 instituições) que juntos participam de quase toda a produção, entretanto há informações explícitas na $\mathrm{WoS}$ do fomento de apenas de 3 países; 
- Há 146 palavras-chave no corpus, as quais agrupamos em 16 termos ligados a conteúdos matemáticos e 62 a aspectos teórico-metodológicos, convergentes com as categorias emergentes de títulos e resumos, que fornecem um panorama do cenário de investigação MTSK na WoS;

- O trabalho mais citado na WoS é um artigo de periódico de impacto na área (CarrilloYañez et al., 2018) em que os criadores do MTSK apresentam o modelo incluindo refinamentos (por exemplo, avanços na categorização) considerando a versão publicada anteriormente em livro (Carrillo et al., 2014).

Nossa principal conclusão é que a produção MTSK é colaborativa e internacional, envolvendo 9 países, na Europa e América Latina. Trata-se de um movimento iberoamericano em rede que contribui para o avanço na disseminação global e, de certo modo, configura-se como um teste de validação internacional do MTSK em diferentes culturas escolares e contextos de prática e formação docente.

Nossos resultados estão limitados apenas aos registros indexados na base de dados Web of Science. Ainda assim, este trabalho materializa parte importante do cenário atual da produção MTSK e dá um primeiro passo no acompanhamento do seu crescimento e desenvolvimento com vistas à análise de impactos na área. Podem ser úteis não somente para grupos e pesquisadores da temática, mas também para formadores que pretendem elaborar cursos e atividades formativas que desenvolvam conhecimento especializado de professores voltados para a prática de ensinar e fazer aprender Matemática. Acreditamos também que os avanços na pesquisa podem ajudar a subsidiar reflexões sobre políticas públicas ligadas à educação e formação docente.

A continuidade do projeto de mapeamento caminhará no sentido de integrar a produção em outras bases de dados com os resultados deste artigo. Com isso poderemos compreender como os resultados deste artigo se comportam frente a outras bases de dados. Mas, para além disso, está em desenvolvimento um estado da arte para aprofundar a perspectiva qualitativa, incluindo a análise das características, alcances, impactos, limites e potencialidades dos avanços científicos vinculados ao MTSK. Para isso, além das categorias analisadas neste artigo, planejamos contemplar: temática dos estudos, outras perspectivas teóricas utilizadas na produção MTSK, objetivos dos trabalhos, conteúdos matemáticos abordados, sujeitos envolvidos na pesquisa, contextos e níveis de ensino contemplados, bem como, principais conclusões e avanços científicos.

\section{Agradecimentos}

Ao fomento da FAPEMAT (Edital Universal 42/2016), apoio do IFMT (Resolução 10/2015/CONSUP) e da Coordenação de Aperfeiçoamento de Pessoal de Nível Superior Brasil (CAPES). Este artigo é uma homenagem ao saudoso amigo Prof. Dr. José Carrillo Yañéz, líder do grupo que criou o marco teórico MTSK. 
DOI: $10.20396 /$ zet.v29i00.8660030

\section{Declaração de contribuição dos autores}

JGMJ concebeu a ideia do presente trabalho, coletou os dados, desenvolveu a metodologia para análise, a implementou e redigiu este artigo.

\section{Declaração de conflito de interesses}

Este autor, JGMJ, declara que participa da Rede Ibero-americana de Investigadores sobre MTSK e não possui conflito de interesses de ordem pessoal, comercial, científica, política ou financeira no manuscrito.

\section{Referências}

Araújo, C. A. (2016). Bibliometria: evolução histórica e questões atuais. Em Questão, 12(12), 11-32.

Arnon, I., Cottrill, J., Dubinsky, E., Oktaç, A., Roa-Fuentes, S., Trigueros, M., \& Weller, K. (2014). APOS theory: A Framework for Research and Curriculum Development in Mathematics Education. New York: Springer. DOI: 10.1007/978-1-4614-7966-6_1

Ashlock, R. B. (2006). Error patterns in computation: Using error patterns to improve instruction. London: Pearson Education.

Ball, D. L., Thames, M. H., \& Phelps, G. (2008). Content Knowledge for Teaching: What Makes It Special? Journal of teacher education, 59(5), 389-407. DOI: $\underline{10.1177 / 0022487108324554}$

Bayoud, J. M. (2011). A comparison of pre-service and experienced elementary teachers' pedagogical content knowledge (PCK) of common error patterns in fractions. [Master's thesis, American University of Beirut]. AUB. DOI: 10938/8658/t-5470.pdf.

Becker, F. (2019). Construção do Conhecimento Matemático: natureza, transmissão e gênese. Bolema: Boletim de Educação Matemática, 33(65), 963-987. DOI: 10.1590/1980-4415v33n65a01

Biembengut, M. S., \& Hein, N. (2007). A resolução de problemas na matemáticas escolar (4 ed.). São Paulo: Contexto.

Campos-Cano, M., \& Flores-Medrano, E. (2019). Prácticas matemáticas: un avance en su caracterización. In J. Carrillo, M. Codes \& L.C. Contreras (Eds.), Actas del IV Congreso Iberoamericano sobre Conocimiento Especializado del Profesor de Matemáticas (pp. 87-94). Huelva: Universidad de Huelva. https://cdn.congresse.me/rlbmixa8vqmkkc7519pjf2p8ex1i

Carrillo, J. (2017). Idiosincrasia del MTSK, investigaciones realizadas y utilidades. In J. Carrillo \& L.C. Contreras (Eds.), Actas del III Jornadas de Investigación en Didáctica de las Matemáticas (pp. 7-10). Huelva: Universidad de Huelva. https://cdn.congresse.me/j2jtromymsv6jzla78f2h533qw67

Carrillo, J., Climent, N., Contreras, L. C., Montes, M. Á., Escudero, D., \& Medrano, E. F. (2014). Un marco teórico para el Conocimiento especializado del Profesor de Matemáticas. Huelva:Universidad de Huelva Publicaciones. 
DOI: $10.20396 /$ zet.v29i00.8660030

Carrillo-Yañez, J., Climent, N., Montes, M., Contreras, L. C., Flores-Medrano, E., Escudero-Ávila, D., ... Muñoz-Catalán, M. C. (2018). The mathematics teacher's specialised knowledge (MTSK) model. Research in Mathematics Education, 20(3), 236-253. DOI: 10.1080/14794802.2018.1479981

Charalambous, C.; Hill, H. C., \& Ball, D. L. (2011). Prospective teachers' learning to provide instructional explanations: how does it look and what might it take? Journal of Mathematics Teacher Education (14), 441-463. DOI: 10.1007/s10857-011-9182-z

Courant, R., \& Robbins, H. (1996). What is mathematics?: an elementery approach to ideias and methods (2 ed.). New York: Oxford University Press.

Crecci, V. M., Nacarato, A. M., \& Fiorentini, D. (2017). Estudos do estado da arte da pesquisa sobre o professor que ensina matemática. Zetetiké, 25(1), 1-6. DOI: 10.20396/zet.v25i1.8649175

Dienes, Z. P. (1975). As seis etapas do processo de aprendizagem em matemática. São Paulo: EPU.

Escudero, D. I., Flores, E., \& Carrillo, J. (2012). El Conocimiento Especializado del Profesor de Matemáticas. Actas del XV Escuela de Invierno en Matemática Educativa (pp. 35-42). México.

Fiorentini, D., Passos, C. L. B., \& Lima, R. C. R. (2016). Mapeamento da pesquisa acadêmica brasileira sobre o professor que ensina Matemática: Período 2001 a 2012. Unicamp. https://www.fe.unicamp.br/pf-fe/pagina_basica/58/e-book-mapeamentopesquisa-pem.pdf

Kaur, B. (2008). Teaching and learning of mathematics: what really matters to teachers and students? ZDM, 40(6), 951-962. DOI: 10.1007/s11858-008-0128-6

Kilpatrick, J., \& Spangler, D. A. (2015). Educating Future Mathematics Education Professors. In L. D. English and D. Kirshner (Eds.), Handbook of International Research in Mathematics Education (3. ed., pp. 297-310). Routledge.

Krulik, S., \& Reys, R. E. (1998). A resolução de problemas na matemáticas escolar (1 ed.). São Paulo: Atual.

Montes, M. A., Contreras, L. C., \& Carrillo, J. (2013). Conocimiento del profesor de matemáticas: Enfoques del MKT y del MTSK. Actas del Investigación en Educación Matemática XVII (pp. 403-410). Bilbao: SEIEM.

Moreira, P. C., \& Ferreira, M. C. C. (2008). A Teoria dos Subconstrutos e o Número Racional como Operador: das estruturas algébricas às cognitivas. Bolema: Boletim de Educação Matemática, 21(31), 103-127.

Moriel Junior, J. G., \& Wielewski, G. D. (2017). Base de conhecimento de professores de matemática: do genérico ao especializado. Revista de Ensino, Educação e Ciências Humanas, 18(2), 126-133. DOI: 10.17921/2447-8733.2017v18n2p126-133

Moriel Junior, J. G., Wielewski, G. D., \& Carrillo, J. (2019). Meta-análise sobre conhecimento para ensinar divisão de frações. Bolema: Boletim de Educação Matemática, 33(65), 988-1026. DOI: 10.1590/1980-4415v33n65a02 
DOI: 10.20396/zet.v29i00.8660030

Paula, E. F., \& Cyrino, M. C. T. d. C. (2017). Identidade profissional de professores que ensinam Matemática: panorama de pesquisas brasileiras entre 2001-2012. Zetetiké, 25(1), 27-45. DOI: 10.20396/zet.v25i1.8647553

Petit, M. M., Laird, R. E., \& Marsden, E. (2010). A focus on fractions: bringing research to the classroom. Routledge.

Policastro, M. S., \& Ribeiro, M. (2021). Conhecimento especializado do professor que ensina matemática relativo ao tópico de divisão. Zetetiké, 29, e021020. DOI: $\underline{10.20396 / z e t . v 29 i 00.8661906}$

Santos, R. N. M., \& Kobashi, N. Y. (2009). Bibliometria, cientometria, infometria: conceitos e aplicações. Pesquisa Brasileira em Ciência da Informação, 2(1), 155-172.

Scheiner, T., Montes, M. A., Godino, J. D., Carrillo, J., \& Pino-Fan, L. R. (2019). What Makes Mathematics Teacher Knowledge Specialized? Offering Alternative Views. International Journal of Science and Mathematics Education, 17(1), 153-172. DOI: $\underline{10.1007 / \mathrm{s} 10763-017-9859-6}$

Shulman, L. S. (1986). Those who understand: Knowledge growth in teaching. Educational researcher, 15(2), 4-14. http://www.jstor.org/stable/1175860

Tardif, M. (2002). Saberes docentes e formação profissional. Petrópolis: Petrópolis: Vozes.

Vasco, D., Moriel Junior, J. G., \& Contreras, L. C. (2017). Subdominios KoT y KSM del Mathematics Teacher's Specialised Knowledge (MTSK): definición, categorías y ejemplos. In J. Carrillo \& L.C. Contreras (Eds.), Actas del III Jornadas de Investigación en Didáctica de las Matemáticas (pp. 29-37). Huelva: Universidad de Huelva. https://cdn.congresse.me/j2jtromymsv6jzla78f2h533qw67 


\section{APÊNDICE}

Tabela. Resumo dos registros da produção MTSK na Web of Science por ano (2013 a 2020)

\begin{tabular}{|c|c|}
\hline Ano & \\
\hline 2013 & ores, Escudero \& Aguilar, 2013; Garcia \& Gonzalez, 2013; Montes, Contreras \& Carrillo, 2013) \\
\hline 2014 & Carrillo, 2014; Escudero-Dominguez \& Carrillo, 2014; Moriel Junior \& Carrillo, 2014) \\
\hline 2015 & $\begin{array}{l}\text { (Escudero-Avila et al., 2015; Liñan, Montes \& Contreras, 2015; Montes \& Carrillo, 2015; Montes et al., } \\
\text { 2015a; Montes et al., 2015b; Vasco et al., 2015) }\end{array}$ \\
\hline 2016 & $\begin{array}{l}\text { suilar, Carrillo \& Munoz-Catalan, 2016; Barrera et al., 2016; Climent et al., 2016; Espinoza-Vazquez } \\
\text { l., 2016) }\end{array}$ \\
\hline 2017 & $\begin{array}{l}\text { (Escudero-Avila, Flores-Medrano \& Carrillo, 2017; Espinoza-Vasquez, Zakaryan \& Yanez, 2017; } \\
\text { Pascual e Montes, 2017) }\end{array}$ \\
\hline 2018 & $\begin{array}{l}\text { (Aguilar-Gonzalez et al., 2018; Badillo \& Fernandez, 2018; Cardenas \& Caceres, 2018; Carrillo-Yañez } \\
\text { et al., 2018; Contreras, Carrillo \& Climent, 2018; Espinoza-Vasquez, Zakaryan \& Yanez, 2018; } \\
\text { Huincahue, Borromeo-Ferri \& Mena-Lorca, 2018; Montes, Contreras \& Carrillo, 2018; Oliveros et al., } \\
\text { 2018; Vasco Mora \& Climent Rodríguez, 2018) }\end{array}$ \\
\hline 2019 & (Carreno \& Climent, 2019; Prieto-González \& Aguilar-González, 2019; Zakaryan \& Ribeiro, 2019) \\
\hline 2020 & $\begin{array}{l}\text { (Carvajal, Martinez \& Soto, 2020; Delgado-Rebolledo \& Zakaryan, 2020; Pizarro, Belmonte \& Arteaga- } \\
\text { Martinez, 2020) }\end{array}$ \\
\hline
\end{tabular}

\section{Lista de registros conforme Web of Science (ordem alfabética)}

Aguilar, A., Carrillo, J., \& Munoz-Catalan, M. C. (2016). Relationships between beliefs and Specialised knowledge (MTSK) about classification of plane figures. In C. Fernández, J. L. González, F. J. Ruiz, T. Fernández \& A. Berciano (Eds.), Investigacion En Educacion Matematica XX (pp. 553-553). Málaga: SEIEM.

Aguilar-Gonzalez, A., Munoz-Catalan, C., Carrillo-Yanez, J., \& Rodriguez-Muniz, L. J. (2018). How to establish connections between specialized knowledge and mathematics teachers' beliefs? PNA. Revista de Investigación en Didáctica de la Matemática, 13(1), 41-61.

Badillo, E., \& Fernandez, C. (2018). Opportunities that emerge from the relationship between perspectives: analysis of knowledge and/or teaching competence. Em L. J. Rodriguez-Muniz, L. Muniz-Rodriguez, A. Aguilar-Gonzalez, P. Alonso, F. J. G. Garcia \& A. Bruno (Eds.), Investigacion En Educacion Matematica XXII (pp. 66-80). Gijón: SEIEM.

Barrera, V. J., Linan, M. M., Munoz-Catalan, C., \& Contreras, L. C. (2016). Specialised knowledge which is mobilised and emerges in a Primary school class when dealing with the relative positions of straight lines. In C. Fernández, J. L. González, F. J. Ruiz, T. Fernández \& A. Berciano (Eds.), Investigacion En Educacion Matematica XX (pp. 167-176). Málaga: SEIEM.

Cardenas, J. A., \& Caceres, M. J. (2018). Exploring conceptions of students to be early childhood teachers about mathematics teachers' professional knowledge. In L. J. Rodriguez-Muniz, L. Muniz-Rodriguez, A. Aguilar-Gonzalez, P. Alonso, F. J. G. Garcia \& A. Bruno (Eds.), Investigacion En Educacion Matematica XXII (pp. 191-200). Gijón: SEIEM.

Carreno, E., \& Climent, N. (2019). Pre-service Secondary Mathematics Teachers' Specialized Knowledge. A Study of the Definitions of Quadrilaterals. PNA. Revista de Investigación en Didáctica de la Matemática, 14(1), 23-53.

Carrillo, J. (2014). Student primary teachers' knowledge (TEDS-M Spain) from the perspective of their specialization. In M. T. González, M. Codes, D. Arnau \& T. Ortega (Eds.), Investigacion En Educacion Matematica XVIII (pp. 115-123). Salamanca: SEIEM. 
Carrillo-Yañez, J., Climent, N., Montes, M., Contreras, L. C., Flores-Medrano, E., Escudero-Ávila, D., ...Muñoz-Catalán, M. C. (2018). The mathematics teacher's specialised knowledge (MTSK) model. Research in Mathematics Education, 20(3), 236-253.

Carvajal, C. A., Martinez, P. F., \& Soto, G. V. (2020). Specialized knowledge of the prospective teachers of mathematics on the logical and syntactic aspects of the mathematical proof. PNA. Revista de Investigación en Didáctica de la Matemática, 14(2), 85-117.

Climent, N., Montes, M. A., Contreras, L. C., Carrillo, J., Linan, M. M., Munoz-Catalan, M. C., ... Leon, F. (2016). Building knowledge of features of learning mathematics by analyzing videos. Avances de Investigación en Educación Matemática, 9, 85-103.

Contreras, L. C., Carrillo, J., \& Climent, N. (2018). Approaching to a student teacher's specialised knowledge from a narrative. In L. J. Rodriguez-Muniz, L. Muniz-Rodriguez, A. AguilarGonzalez, P. Alonso, F. J. G. Garcia \& A. Bruno (Eds.), Investigacion En Educacion Matematica XXII (pp. 51-65). Gijón: SEIEM.

Delgado-Rebolledo, R., \& Zakaryan, D. (2020). Relationships Between the Knowledge of Practices in Mathematics and the Pedagogical Content Knowledge of a Mathematics Lecturer. International Journal of Science and Mathematics Education, 18(3), 567-587.

Escudero-Avila, D., Flores-Medrano, E., \& Carrillo, J. (2017). Describing a secondary mathematics teacher's specialised knowledge of functions. In Proceedings of the Tenth Congress of the European Society for Research in Mathematics Education (pp. 3280-3287).

Escudero-Avila, D. I., Carrillo, J., Flores-Medrano, E., Climent, N., Contreras, L. C., \& Montes, M. (2015). Mathematics Teacher's Specialised Knowledge Detected in the Circle Chord Problem Solution. PNA. Revista de Investigación en Didáctica de la Matemática, 10(1), 53-77.

Escudero-Dominguez, A., \& Carrillo, J. (2014). Mathematical knowledge of quadrilateral in prospective primary teachers. In M. T. González, M. Codes, D. Arnau \& T. Ortega (Eds.), Investigacion En Educacion Matematica XVIII (pp. 267-276). Salamanca: SEIEM.

Espinoza-Vasquez, G., Zakaryan, D., \& Yanez, J. C. (2017). Use of analogies in teaching the concept of function: Relation between knowledge of topics and knowledge of mathematics teaching. In Proceedings of the Tenth Congress of the European Society for Research in Mathematics Education (pp. 3288-3295).

Espinoza-Vasquez, G., Zakaryan, D., \& Yanez, J. C. (2018). The Mathematics Teacher's Specialized Knowledge in the Use of Analogy on Teaching the Function Concept. Revista Latinoamericana De Investigacion En Matematica Educativa-Relime, 21(3), 301-324.

Espinoza-Vazquez, G., Verdugo-Hernandez, P., Zakaryan, D., Carrillo, J. e Montoya-Delgadillo, E. (2016). Toward a relationship between MWS and MTSK through the function concept. In C. Fernández, J. L. González, F. J. Ruiz, T. Fernández \& A. Berciano (Eds.), Investigacion En Educacion Matematica XX (pp. 197-206). Málaga: SEIEM.

Flores, E., Escudero, D. I., \& Aguilar, A. (2013). Opportunities offered by some scenarios for showing MTSK' evidences. In A. Berciano, G. Gutiérrez, A. Estepa \& N. Climent (Eds.), Investigacion En Educacion Matematica XVII (pp. 275-282). Bilbao, Espanha: SEIEM.

Garcia, M. D. L., \& Gonzalez, L. C. C. (2013). Strengths and weaknesses in Geometry Knowledge of Topics (KoT) of Prospective Primary Teachers. In A. Berciano, G. Gutiérrez, A. Estepa \& N. Climent (Eds.), Investigacion En Educacion Matematica XVII (pp. 337-344). Bilbao: SEIEM.

Huincahue, J., Borromeo-Ferri, R., \& Mena-Lorca, J. (2018). Math modeling knowledge from reflection in math teachers initial training. Enseñanza de las Ciencias, 36(1), 99-115. 
DOI: $10.20396 /$ zet.v29i00.8660030

Liñan, M. M., Montes, M. A., \& Contreras, L. C. (2015). Knowledge about straight line of a third grade primary teacher. In C. Fernández, M. Molina \& N. Planas (Eds.), Investigacion En Educacion Matematica XIX (pp. 335-342). Alicante: SEIEM.

Montes, M., \& Carrillo, J. (2015). What does it mean as a teacher to "know infinity"? The case of convergence series. In K. Krainer e N. Vondrova (Eds.), Proceedings of the Ninth Conference of the European Society for Research in Mathematics Education (pp. 3220-3226).

Montes, M., Contreras, L. C., \& Carrillo, J. (2018). Teacher, which is the biggest number? Transcending school curriculum in the exploration of teachers' specialized knowledge. Avances de Investigación en Educación Matemática, 13, 5-20.

Montes, M., Escudero-Avila, D., Flores-Medrano, E., Munoz-Catalan, M. C., \& Carrillo, J. (2015a). The forum as a context for exploring in-service teachers' professional knowledge. In C. Fernández, M. Molina \& N. Planas (Eds.), Investigacion En Educacion Matematica XIX (pp. 381-389). Alicante: SEIEM.

Montes, M. A., Contreras, L. C., \& Carrillo, J. (2013). Knowledge of the Mathematics' Teacher: Approaches of MKT and MTSK. In A. Berciano, G. Gutiérrez, A. Estepa \& N. Climent (Eds.), Investigacion En Educacion Matematica XVII (pp. 403-410). Bilbao: SEIEM.

Montes, M. A., Contreras, L. C., Linan, M. D., Munoz-Catalan, M. C., Climent, N., \& Carrillo, J. (2015b). Arithmetic Knowledge of prospective teachers. Strengths and Weaknesses. Revista De Educacion(367), 36-62. DOI: 10.4438/1988-592x-re-2015-367-282

Moriel Junior, J. G., \& Carrillo, J. (2014). Exploring indications of specialised knowledge for mathematics teaching through application of the MTSK model. In M. T. González, M. Codes, D. Arnau \& T. Ortega (Eds.), Investigacion En Educacion Matematica XVIII (pp. 465-474). Salamanca: SEIEM.

Oliveros, I., Pascual, M. I., Codes, M., \& Martin, J. P. (2018). Knowledge of the practice of mathematics shared between prospective teachers through video analysis. In L. J. RodriguezMuniz, L. Muniz-Rodriguez, A. Aguilar-Gonzalez, P. Alonso, F. J. G. Garcia \& A. Bruno (Eds.), Investigacion En Educacion Matematica XXII (pp. 407-416). Gijón: SEIEM.

Pascual, M. I., \& Montes, M. (2017). Questions of in-service teachers as a guideline of their instruction: a metacognitive approach. In J.M. Muñoz-Escolano, A. Arnal-Bailera, P. BeltránPellicer, M. L. Callejo \& J. Carrillo (Eds.), Investigacion En Educacion Matematica XXI (pp. 387-395). Zaragoza: SEIEM.

Pizarro, N., Belmonte, J. M., \& Arteaga-Martinez, B. (2020). A Didactic Analysis of the Classroom Practice in the Teaching of Analogue Clock Reading. Educacion Xxl, 23(1), 409-436.

Prieto-González, A. P., \& Aguilar-González, A. A. (2019). MTSK in the initial training of teachers in early childhood education for the design of activities. Edma 0-6-Educacion Matematica En La Infancia, 8(2), 109-133.

Vasco, D., Climent, N., Escudero-Avila, D., \& Flores-Medrano, E. (2015). The characterisation of the specialised knowledge of a university lecturer in linear algebra. In K. Krainer \& N. Vondrova (Eds.), Proceedings of the Ninth Conference of the European Society for Research in Mathematics Education (pp. 3283-3288).

Vasco Mora, D. L., \& Climent Rodríguez, N. (2018). The Study of the Specialized Knowledge of two Linear Algebra Lecturers. PNA. Revista de Investigación en Didáctica de la Matemática, 12(3), 129-146.

Zakaryan, D., \& Ribeiro, M. (2019). Mathematics teachers' specialized knowledge: a secondary teacher's knowledge of rational numbers. Research in Mathematics Education, 21(1), 25-42. 